\title{
Energy Stable Linear Schemes for Mass-Conserved Gradient Flows with Peng-Robinson Equation of State
}

\author{
Qiujin Peng ${ }^{1,2}$, Hongwei $\mathrm{Li}^{3, *}$ and Zuoliang $\mathrm{Xu}^{4}$ \\ ${ }^{1}$ School of Mathematics and Statistics, Xuchang University, Xuchang, \\ 461000, P.R. China. \\ ${ }^{2}$ Institute for Mathematical Sciences, Renmin University of China, Beijing, \\ 100872, P.R. China. \\ ${ }^{3}$ School of Mathematics and Statistics, Shandong Normal University, Jinan, \\ 250014, P.R. China. \\ ${ }^{4}$ School of Information, Renmin University of China, Beijing, 100872, P.R. China.
}

Received 14 April 2018; Accepted (in revised version) 12 May 2018.

\begin{abstract}
First and second order numerical schemes for the fourth order parabolic equation with Peng-Robinson equation of state, which are based on recently proposed invariant energy quadratisation method are developed. Both schemes are linear, unconditionally energy stable and uniquely solvable. The reduced linear systems are symmetric and positive definite, so that their solutions can be efficiently found. Numerical results demonstrate the good performance of the schemes, consistent with experimental data.
\end{abstract}

AMS subject classifications: 65N30, 65N50, 49S05

Key words: Conservative gradient flow, Peng-Robinson equation of state, invariant energy quadratisation, unconditional energy stability.

\section{Introduction}

Modeling and simulation of the multi-phase hydrocarbon systems of the oil-exploitation processes are popular in engineering practice [17]. The subsurface crude oil reservoirs often contain small amounts of nitrogen, carbon dioxide and hydrocarbons from methane through $\mathrm{C}_{30+}$ in both vapor phase and liquid phase together with solid phase (rock or soil) and water phase $[29,41]$. The anisotropic forces at the surface or interface between immiscible and/or partially miscible fluids at pore-scale yield the surface tension. The capillary effect at Darcy-scale and the resultant capillary pressure caused by the surface tension is one of the major forces in fluid flow and transport in subsurface reservoirs. It is also the leading mechanism in oil recovery from fractured oil reservoirs [29]. In addition, the surface tension influences the relative permeability and residual saturation in porous medium

*Corresponding author. Email addresses: pengqiuj in@ruc .edu.cn (Q. Peng), hwli@sdnu.edu.cn (H. Li), xuzl@ruc.edu.cn (Z. Xu) 
processes and thus influences the flow and the transport of the vapor and liquid phases on the pore scale. Therefore, appropriate mathematical models play an important role in simulation and prediction of interface phenomena and in the calculation of important interface parameters connected with different phases of oil mixture.

There are at least three main approaches to the study of interface phenomena. The first one is carried out either by molecular Monte Carlo simulations or molecular dynamics simulation with an intermolecular potential function $[12,38]$. The second approach is known as sharp interface modeling. In this case, the interface is considered as a zero-thickness two-dimensional entity, where density experiences a big jump [23,28,38]. Although it can predict the shape and the dynamics of the interface with a given interface tension, the interface details can not be derived. The third one is the phase field model [44], gradient theory [4], diffuse interface model [33]. The density is constant for homogeneous phase and experiences continuous variation at the interface.

In our simulations of the interface phenomena, the phase field model is selected. This well-known model was established by Van der Waals [42] in his work on the interface predictions based on thermodynamic principles [1], and extended by Cahn and Hilliard [5-7]. To describe the final steady state of a system based on the well accepted second thermodynamic law, the Helmholtz free energy is usually used as starting point. According to the phase field model, the total Helmholtz free energy often consists of the homogeneous part of pure phase for substance and the gradient part determined by the density variation at the interface. Thus the total energy can be approximated by a functional, describing the phenomenological characters of the investigated system - e.g. by the double-well potential $[21,22]$, the molecular beam epitaxy model with [39] or without $[8,9,40]$ slope selection, the Ericksen-Leslie model for nematic liquid crystal flows [13], the phase field crystal energy functional $[2,24]$, the energy of polymer hydrogels $[37,50]$ and so on. On the other hand, to provide reliable predictions for all thermodynamic properties, the total Helmholtz free energy has been obtained from the equation of state for a real substance. To derive the corresponding free energy expressions, serious efforts are needed. In particular, for every substance of interest at each development step, some practical laboratory experiments should be carried out along with the study of the interaction of different components or different phases for real mixture. The Peng-Robinson equation of state model is used more often than other cubic equations of the state due to better prediction of fluid densities and reliable results in the vicinity of critical regions [34,41].

These energy functionals are used to derive the Euler-Lagrange equation for the system equilibrium. The popular Allen-Cahn and Cahn-Hilliard equations are typical examples of such flows. A variety of numerical schemes for parabolic equations are based on these energy functionals. Note that the main concern for these methods is the energy stability and, excluding the approach [40], there are four ways to guarantee this. The first one, relying on the addition to discrete equation of a stabilised term of order $\mathscr{O}(\Delta t)$ or higher, is called the stabilised method [44,51]. The stabilised parameter should be expedient to balance the stability and convergence of the scheme [31]. The second one is the convexsplitting scheme proposed by Eyre [15] for the Cahn-Hilliard equation. The basic idea of this method is that if the total energy could be represented as the difference of two con- 
vex functions of the target variable. Then the derivatives of the minuend and subtractive terms are, respectively, derived implicitly and explicitly. The uniqueness of this scheme is based on the fact that the corresponding solution is the minimiser of the modified convex total energy. This method has been applied to almost all above mentioned models cf. Refs. $[2,3,8,18,20,24,43,46,49,52]$ and the references therein. The third approach is called the exponential time difference (ETD) method and involves exact integration of the linear part of the governing equations along with the approximation of the integral for nonlinear terms [11]. This method was used to solve semilinear parabolic equations [25] and phase field elastic bending energy models [48]. The fourth one is the newly introduced invariant energy quadratisation (IEQ) method. It has a number of advantages such as the linearity, unconditional energy stability and accuracy. The method has been applied to the Cahn-Hilliard type equation with various energy functionals [19], the binary fluidsurfactant phase field model [54], the phase field crystal model [53], phase field dendritic crystal growth model [56], second order parabolic equations with the Peng-Robinson equation of state [30].

The equations derived from the Peng-Robinson equation of state contain logarithmic and fractional singular terms. Various problems for such equations have been studied. Thus Kou and Sun [27] presented the solvability conditions for the Euler-Lagrange equation in multi-component case and established the maximum principle for the weighted molar density. Moreover, approximate solutions of these equations have been obtained by modified Newton and finite element methods. The single component [38] and multicomponent [16] second order parabolic equation with mass conservation restriction have been solved using a first order convex-splitting scheme. Besides, numerical solution of the naturally mass conservative fourth order parabolic equation with Peng-Robinson equation of state for single- and multi-component cases was also obtained. In the single-component case, the energy stability, unique solvability and $l^{\infty}$ convergence of a first order convexsplitting scheme [35] and two second order schemes [36] have been established. In the multi-component case, the relations between different components are decoupled and the solution of the new system of equations was derived by a semi-implicit unconditionally stable scheme combined with a mixed finite element method.

In this work, the mass-conserved gradient flow with Peng-Robinson equation for the state of particular pure substances is solved by IEQ based Crank-Nicolson schemes of the first and second orders. The paper is organised as follows. In Sections 2 and 3, the corresponding fourth order parabolic equation and its modified version are introduced. Section 4 deals with numerical schemes and the unconditional energy stability. The numerical results presented in Section 5, demonstrate the efficiency and effectiveness of these schemes and concluding remarks are in Section 6.

\section{Mathematical Model of Peng-Robinson Equation of State}

Let $\mathbf{x}$ and $t$ refer to spacial and time variables, respectively. By $n=n(\mathbf{x}, t)$ we denote any special hydrocarbon substance in a real crude oil mixture. The total Helmholtz free energy of this substance in the oil recovery process, can be represented via the Peng-Robinson 
equation of state [26] as follows

$$
F(n(\mathbf{x}, t))=\int_{\Omega}\left[f_{0}(n(\mathbf{x}, t))+\frac{c}{2}|\nabla n(\mathbf{x}, t)|^{2}\right] d \mathbf{x},
$$

where the homogeneous Helmholtz free energy $f_{0}(n)$ of a single component fluid has the form

$$
\begin{aligned}
& \begin{array}{l}
f_{0}(n)=f_{0}^{\text {ideal }}(n)+f_{0}^{\text {excess }}(n) \\
\quad=f_{0}^{\text {ideal }}(n)+f_{01}^{\text {excess }}(n)+f_{02}^{\text {excess }}(n),
\end{array} \\
& f_{0}^{\text {ideal }}(n)=R T n(\ln n-1), \\
& f_{01}^{\text {excess }}(n)=-n R T \ln (1-b n), \\
& f_{02}^{\text {excess }}(n)=\frac{a(T) n}{2 \sqrt{2} b} \ln \left(\frac{1+(1-\sqrt{2}) b n}{1+(1+\sqrt{2}) b n}\right) .
\end{aligned}
$$

Here, $R \approx 8.31432 \mathrm{JK}^{-1} \mathrm{~mol}^{-1}$ is the universal gas constant and $T$ the substance temperature. The parameters $a(T)$ and $b$ are described in Appendix.

Mathematically the problem can be formulated as follows: Find an $n^{*} \in H$ such that

$$
F\left(n^{*}\right)=\min _{n \in \mathbb{H}} F(n)
$$

subject to the constrain

$$
\int_{\Omega} n d \mathbf{x}=N
$$

where $\mathbb{H}$ is a space of sufficiently smooth functions. We note that $N$ represents the fixed amount of material mass.

Using variational methods, one can show that $n$ satisfies the initial value problem for a fourth order parabolic equation — viz.

$$
\begin{aligned}
& \frac{\partial n(\mathbf{x}, t)}{\partial t}=\nabla \cdot \nabla \frac{\delta F}{\delta n}(n)=-c \Delta^{2} n(\mathbf{x}, t)+\Delta \mu_{0}(n(\mathbf{x}, t)), \\
& n(\mathbf{x}, 0)=n_{0}(\mathbf{x}) .
\end{aligned}
$$

The function $\mu_{0}(n(\mathbf{x}, t))$ represents the first order variational derivative of the total homogeneous energy $\int_{\Omega} f_{0}(n(\mathbf{x}, t)) d \mathbf{x}$ with respect to the molar density $n(\mathbf{x}, t)$. It has the form

$$
\mu_{0}(n)=R T \ln \left(\frac{n}{1-b n}\right)+\frac{R T b n}{1-b n}+\frac{a(T)}{2 \sqrt{2} b} \ln \left(\frac{1+(1-\sqrt{2}) b n}{1+(1+\sqrt{2}) b n}\right)-\frac{a(T) n}{1+2 b n-b^{2} n^{2}}
$$

and can be considered as the homogeneous chemical potential of the substance.

Two types of boundary conditions are considered in this work:

1. All variables are periodic. 
2. Function $n$ satisfies the zero boundary flux condition - i.e.

$$
\left.\partial_{\mathbf{n}} n\right|_{\partial \Omega}=\left.\nabla \mu \cdot \mathbf{n}\right|_{\partial \Omega}=0
$$

where $\mathbf{n}$ is the unit outward normal on the boundary $\partial \Omega$.

The mass conservation and the energy stability of such problems have been considered earlier [36] and are used here without any comments.

Remark 2.1. The so-called convex splitting approach used to discretise the nonlinear term while preserving unconditional energy stability leads to nonlinear schemes, the implementations of which is complicated and the computational cost is high. Therefore, our goal here is to develop efficient linear schemes for fourth order parabolic equations based on IEQ approach.

\section{Modified Total Free Energy and Transform System}

The key point in IEQ approach is to make the homogeneous Helmholtz free energy quadratic by changing auxiliary variables. A particularly significant property of the free energy is the boundedness from below.

The homogeneous part $f_{0}(n)$ of the total free energy will be written in the form $f_{0}(n)=$ $f_{01}(n)+f_{02}(n)$, where

$$
\begin{aligned}
& f_{01}(n)=f_{0}^{\text {ideal }}(n)+f_{01}^{\text {excess }}(n)=R T n(\ln n-1)-n R T \ln (1-b n), \\
& f_{02}(n)=f_{02}^{\text {excess }}(n)=\frac{a(T) n}{2 \sqrt{2} b} \ln \left(\frac{1+(1-\sqrt{2}) b n}{1+(1+\sqrt{2}) b n}\right) .
\end{aligned}
$$

The function $f_{01}(n)$ is singular at the end points of its physically reasonable region $(0,1 / b)$. To remove its singularities at $n=0$ and $n=1 / b$, we replace it by a $C^{2}$ continuous, convex, piecewise function $\tilde{f}_{01}(n)$, with the reasonable domain $(-\infty,+\infty)$. For an $\epsilon \in(0,1 / 2 b)$, the modified version of $f_{01}(n)$ has the form

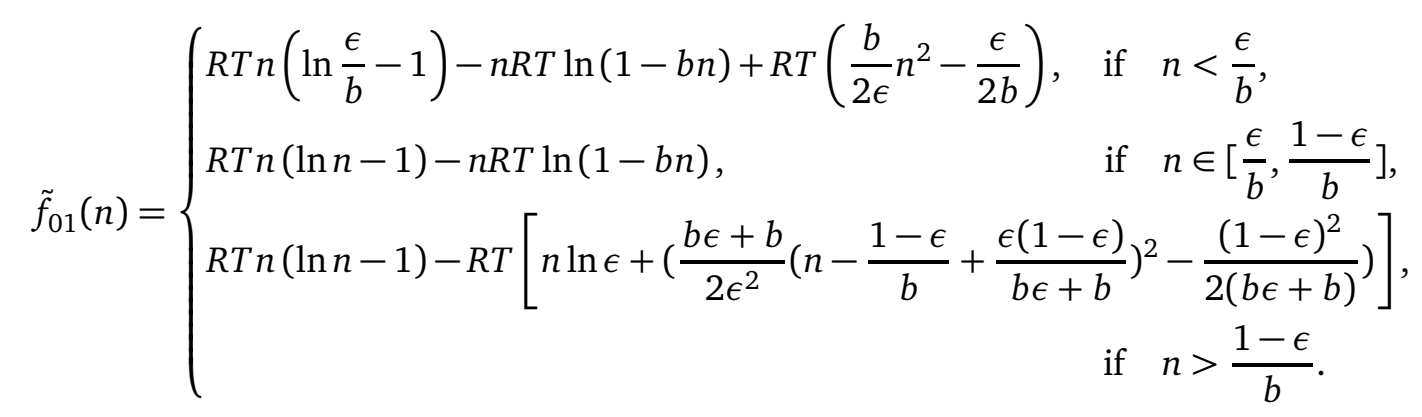

In the interval $(0,1 / b)$, the error of approximation $f_{01}(n)$ by $\tilde{f}_{01}(n)$ does not exceed a constant multiple of $\epsilon-\mathrm{cf}$. $[10,14]$.

For the function $f_{02}(n)$, the physically reasonable region $(0,1 / b)$ is included in its functional domain $(1-\sqrt{2}, 1+\sqrt{2})$. We will carry out the computations in the interval 
$[1-\sqrt{2}+\varepsilon, 1+\sqrt{2}-\varepsilon]$, where $\varepsilon$ is a small positive number, so that the function $f_{02}(n)$ is bounded from below during the whole procedure.

The modified homogeneous Helmholtz free energy of the single component fluid takes the form $\tilde{f}_{0}(n)=\tilde{f}_{01}(n)+f_{02}(n)$. The function $\tilde{f}_{0}(n)$ is bounded from below but may take non-positive values at some points in the domain. Thus

$$
\tilde{F}_{\text {tot }}(n)=\int_{\Omega}\left[\frac{c}{2}|\nabla n|^{2}+\tilde{f}_{0}(n)+B-B\right] d x,
$$

where $B$ is a positive constant such that $\tilde{f}_{0}(n)+B>0$. Using the notation

$$
W=\sqrt{\tilde{f}_{0}(n)+B}
$$

we rewrite the total free energy (3.1) as

$$
\tilde{F}_{\text {tot }}(n, W)=\int_{\Omega}\left[\frac{c}{2}|\nabla n|^{2}+W^{2}-B\right] d x .
$$

Variational methods applied to the Eq. (3.2) produce a new equivalent system

$$
\begin{aligned}
& n_{t}=\Delta \mu, \\
& \mu=-c \Delta n+W H(n), \\
& W_{t}=\frac{1}{2} H(n) n_{t},
\end{aligned}
$$

where $H(n)=\tilde{f}_{0}^{\prime}(n) / \sqrt{\tilde{f}_{0}(n)+B}$ and $\tilde{f}_{0}^{\prime}(n)=\left(\delta \tilde{f}_{0} / \delta n\right)(n)$. The Eqs. (3.3)-(3.5) form a closed PDE system with the initial conditions

$$
n(t=0)=n_{0}, \quad \mu(t=0)=\mu\left(n_{0}\right), \quad W(t=0)=\sqrt{\tilde{f}_{0}\left(n_{0}\right)+B} .
$$

Considering the inner product

$$
(h(\mathbf{x}), g(\mathbf{x})):=\int_{\Omega} h(\mathbf{x}) g(\mathbf{x}) d \mathbf{x}
$$

on the space $L^{2}$ and denoting by $\|\cdot\|$ the corresponding norm, we compute the sum of the inner products of (3.3)-(3.5) with $-\mu, n_{t}$ and $W$, thus obtaining the energy dissipation law for (3.3)-(3.5) - viz.

$$
\frac{d \tilde{F}_{\text {tot }}(n, W)}{d t}=-\|\nabla \mu\|^{2} \leq 0 .
$$

\section{Numerical Schemes}

Here we want to develop semi-discrete time marching numerical schemes for the system (3.3)-(3.5) and establish their unconditionally energy stability. Let $\delta t>0$ be the time step size and $t^{k}:=k \delta t$ for $0 \leq k \leq K$ with the ending time $T=K \delta t$. 


\subsection{A first order scheme}

Assuming that $\left\{n^{k}, \mu^{k}, W^{k}\right\}$ are already known, we determine the terms $\left\{n^{k+1}, \mu^{k+1}\right.$, $\left.W^{k+1}\right\}$ from the first order temporal semi-discretised system

$$
\begin{aligned}
& \frac{n^{k+1}-n^{k}}{\delta t}=\Delta \mu^{k+1}, \\
& \mu^{k+1}=-c \Delta n^{k+1}+W^{k+1} H\left(n^{k}\right), \\
& \frac{W^{k+1}-W^{k}}{\delta t}=\frac{1}{2} H\left(n^{k}\right) \frac{n^{k+1}-n^{k}}{\delta t}
\end{aligned}
$$

provided with a periodic (or no-flux) boundary condition.

It follows from (4.3) that

$$
W^{k+1}=W^{k}+\frac{1}{2} H\left(n^{k}\right)\left(n^{k+1}-n^{k}\right)
$$

Here and hereafter, we write $W^{k}$ for $W\left(n^{k}\right)$. Substituting (4.4) into (4.2) yields

$$
\mu^{k+1}=-c \Delta n^{k+1}+H\left(n^{k}\right)\left[W^{k}+\frac{1}{2} H\left(n^{k}\right)\left(n^{k+1}-n^{k}\right)\right],
$$

and using the representation (4.5) in (4.1), we obtain

$$
n^{k+1}=n^{k}+\delta t \Delta\left\{-c \Delta n^{k+1}+H\left(n^{k}\right)\left[W^{k}+\frac{1}{2} H\left(n^{k}\right)\left(n^{k+1}-n^{k}\right)\right]\right\} .
$$

Therefore, we can now determine $n^{k+1}$ from (4.6) and then compute $\mu^{k+1}$ and $W^{k+1}$. Note that the introduction of the auxiliary variable $W$ comes at no extra computational cost.

Theorem 4.1. The linear equation (4.6) is symmetric (self-adjoint) and positive definite for the variable $n^{k+1}$.

Proof. Considering the inner products of $n^{k}$ and 1, we have

$$
\int_{\Omega} n^{k+1} d x=\int_{\Omega} n^{k} d x=\cdots=\int_{\Omega} n^{0} d x
$$

Set $\alpha_{n}^{0}:=(1 /|\Omega|) \int_{\Omega} n^{0} d x$ and let

$$
\beta^{\mu}=\frac{1}{|\Omega|} \int_{\Omega} \mu^{k+1} d x, \quad \hat{n}^{k+1}=n^{k+1}-\alpha_{n}^{0}, \quad \hat{\mu}^{k+1}=\mu^{k+1}-\beta^{\mu} .
$$

Using (4.1)-(4.3), we note that $n^{k+1}$ and $\mu^{k+1}$ are the solutions of the equations

$$
\begin{aligned}
& \frac{n}{\delta t}-\Delta \mu=\frac{\hat{n}^{k}}{\delta t} \\
& \mu=-c \Delta n+H\left(n^{k}\right) W\left(n^{k}\right)+\frac{H^{2}\left(n^{k}\right)}{2}\left(n-n^{k}\right)-\beta^{\mu}
\end{aligned}
$$


with the term $\mu$ satisfying the condition

$$
\int_{\Omega} u d x=0 .
$$

We define the inverse Laplace operator $\Delta^{-1}$ from the equations

$$
\begin{aligned}
\Delta v & =u \\
\int_{\Omega} u d x & =0
\end{aligned}
$$

with periodic or no-flux boundary conditions.

Applying $-\Delta^{-1}$ to the Eq. (4.8) and taking into account relation (4.9), one obtains

$$
-\frac{\Delta^{-1} n}{\delta t}-c \Delta n+\frac{H^{2}\left(n^{k}\right)}{2} n-\beta^{\mu}=-\frac{\Delta^{-1} \hat{n}^{k}}{\delta t}-H\left(n^{k}\right) W\left(n^{k}\right)+\frac{H^{2}\left(n^{k}\right)}{2} n^{k} .
$$

We write this equation as $\operatorname{Ln}=f$. Then for any $u, v$ such that $\int_{\Omega} u d x=\int_{\Omega} v d x=0$, the relations

$$
\begin{aligned}
& (L u, v)=\left(-\frac{\Delta^{-1} u}{\delta t}, v\right)-c(\Delta u, v)+\left(\frac{H^{2}\left(n^{k}\right)}{2} u, v\right)-\left(\beta^{\mu}, v\right), \\
& (L v, u)=\left(-\frac{\Delta^{-1} v}{\delta t}, u\right)-c(\Delta v, u)+\left(\frac{H^{2}\left(n^{k}\right)}{2} v, u\right)-\left(\beta^{\mu}, u\right)
\end{aligned}
$$

hold. Note that $(L u, v)=(L v, u)$, hence $L$ is a self-adjoint linear operator. If $v=u$, then

$$
\begin{aligned}
(L u, u) & =\left(-\frac{\Delta^{-1} u}{\delta t}, u\right)-c(\Delta u, u)+\left(\frac{H^{2}\left(n^{k}\right)}{2} u, u\right)-\left(\beta^{\mu}, u\right) \\
& =\frac{1}{\delta t}\|u\|_{H^{-1}}^{2}+c\|\nabla u\|^{2}+\frac{1}{2}\left\|H\left(n^{k}\right) u\right\|^{2} \geq 0,
\end{aligned}
$$

and the inequality becomes an equality if and only if $u=0$.

Remark 4.1. The well-posedness of the linear equation $L n=f$ can be established via Lax-Milgram theorem. On the set $L_{p e r}^{2}(\Omega)$ we define the inner product and the norm by $(u, v)_{L}:=(L u, v)$ and $\|u\|_{L}:=\sqrt{(L u, u)}$, respectively, where $L_{p e r}^{2}(\Omega)$ denotes the subspace of all functions $u \in L^{2}(\Omega)$ with the periodic boundary condition. Considering the subset

$$
S=\left\{u \in L_{p e r}^{2}(\Omega) ;\|u\|_{L}<\infty\right\}
$$

we note that it is a Hilbert space with respect to the above inner product. Therefore, the Lax-Milgram theorem yields that the Eq. (4.6) has a unique weak solution in $S$.

Let us now consider the stability of this first-order scheme. 
Theorem 4.2. The linear scheme (4.1)-(4.3) is unconditionally energy stable - i.e. it satisfies the discrete dissipation law

$$
F_{1 s t}^{k+1} \leq F_{1 s t}^{k}-\delta t\left\|\nabla \mu^{k+1}\right\|^{2}
$$

where

$$
F_{1 s t}^{k}=\frac{c}{2}\left\|\nabla n^{k}\right\|^{2}+\left\|W^{k}\right\|^{2}
$$

Proof. Considering the $L^{2}$-inner products of (4.1) and $-\mu^{k+1},(4.2)$ and $\left(n^{k+1}-n^{k}\right) /(\delta t)$, (4.3) and $-2 W^{k+1}$, we respectively obtain

$$
\begin{aligned}
\left(\frac{n^{k+1}-n^{k}}{\delta t},-\mu^{k+1}\right)= & \left(\Delta \mu^{k+1},-\mu^{k+1}\right)=\left\|\nabla \mu^{k+1}\right\|^{2} \\
\left(\mu^{k+1}, \frac{n^{k+1}-n^{k}}{\delta t}\right)= & \left(-c \Delta n^{k+1}, \frac{n^{k+1}-n^{k}}{\delta t}\right)+\left(W^{k+1} H\left(n^{k}\right), \frac{n^{k+1}-n^{k}}{\delta t}\right) \\
= & \frac{c}{2 \delta t}\left[\left\|\nabla n^{k+1}\right\|^{2}-\left\|\nabla n^{k}\right\|^{2}+\left\|\nabla\left(n^{k+1}-n^{k}\right)\right\|^{2}\right] \\
& +\left(W^{k+1} H\left(n^{k}\right), \frac{n^{k+1}-n^{k}}{\delta t}\right), \\
\left(\frac{W^{k+1}-W^{k}}{\delta t}, 2 W^{k+1}\right) & =\left(\frac{1}{2} H\left(n^{k}\right) \frac{n^{k+1}-n^{k}}{\delta t}, 2 W^{k+1}\right) .
\end{aligned}
$$

Note that the identity $2(a-b, a)=|a|^{2}-|b|^{2}+|a-b|^{2}$ was used in the Eq. (4.12). Besides, the Eq. (4.13) implies

$$
-\frac{1}{\delta t}\left(\left\|W^{k+1}\right\|^{2}-\left\|W^{k}\right\|^{2}+\left\|W^{k+1}-W^{k}\right\|^{2}\right)=-\left(H\left(n^{k}\right) \frac{n^{k+1}-n^{k}}{\delta t}, W^{k+1}\right) .
$$

Taking into account the Eqs. (4.11), (4.12), and (4.14), we arrive at the equation

$$
\begin{aligned}
& \frac{c}{2 \delta t}\left[\left\|\nabla n^{k+1}\right\|^{2}-\left\|\nabla n^{k}\right\|^{2}+\left\|\nabla\left(n^{k+1}-n^{k}\right)\right\|^{2}\right] \\
& \quad+\frac{1}{\delta t}\left[\left\|W^{k+1}\right\|^{2}-\left\|W^{k}\right\|^{2}+\left\|W^{k+1}-W^{k}\right\|^{2}\right]=-\left\|\nabla \mu^{k+1}\right\|^{2},
\end{aligned}
$$

which yields the inequality (4.10).

\subsection{A second order scheme}

We now introduce a second order time stepping scheme of the Crank-Nicolson type for the systems (3.3)-(3.5). Assuming that $\left\{n^{k}, \mu^{k}, W^{k}\right\}$ and $\left\{n^{k-1}, \mu^{k-1}, W^{k-1}\right\}$ are already known, we determine the terms $\left\{n^{k+1}, \mu^{k+1}, W^{k+1}\right\}$ from the second order temporal semidiscretised system

$$
\frac{n^{k+1}-n^{k}}{\delta t}=\Delta \mu^{k+1 / 2}
$$


Stable Linear Schemes for Peng-Robinson EoS

$$
\begin{aligned}
& \mu^{k+1 / 2}=-c \Delta n^{k+1 / 2}+W^{k+1 / 2} H\left(n^{*}\right), \\
& \frac{W^{k+1}-W^{k}}{\delta t}=\frac{1}{2} H\left(n^{*}\right) \frac{n^{k+1}-n^{k}}{\delta t},
\end{aligned}
$$

where

$$
n^{k+1 / 2}=\frac{n^{k+1}+n^{k}}{2}, \quad n^{*}=\frac{3}{2} n^{k}-\frac{1}{2} n^{k-1}, \quad W^{k+1 / 2}=\frac{W^{k+1}+W^{k}}{2}, \quad \mu^{k+1 / 2}=\frac{\mu^{k+1}+\mu^{k}}{2} .
$$

The Eq. (4.17) can be rewritten as

$$
W^{k+1}=W^{k}+\frac{H\left(n^{*}\right)}{2}\left(n^{k+1}-n^{k}\right)
$$

and adding $W^{k}$ to (4.18) yields

$$
W^{k+1 / 2}=\frac{W^{k+1}+W^{k}}{2}=W^{k}+\frac{H\left(n^{*}\right)}{4}\left(n^{k+1}-n^{k}\right)
$$

It follows from (4.15), (4.16) and (4.19) that

$$
\begin{aligned}
\frac{n^{k+1}-n^{k}}{\delta t} & =-c \Delta^{2} n^{k+1 / 2}+\Delta\left\{\left[W^{k}+\frac{H\left(n^{*}\right)}{4}\left(n^{k+1}-n^{k}\right)\right] H\left(n^{*}\right)\right\} \\
& =-\frac{c}{2} \Delta^{2} n^{k+1}-\frac{c}{2} \Delta^{2} n^{k}+\Delta\left[\frac{H^{2}\left(n^{*}\right)}{4} n^{k+1}\right]+\Delta\left[W^{k} H\left(n^{*}\right)-\frac{H^{2}\left(n^{*}\right)}{4} n^{k}\right]
\end{aligned}
$$

or

$$
\frac{n^{k+1}}{\delta t}+\frac{c}{2} \Delta^{2} n^{k+1}-\frac{1}{4} \Delta\left[H^{2}\left(n^{*}\right) n^{k+1}\right]=\frac{n^{k}}{\delta t}-\frac{c}{2} \Delta^{2} n^{k}+\Delta\left[W^{k} H\left(n^{*}\right)-\frac{H^{2}\left(n^{*}\right)}{4} n^{k}\right] .
$$

Theorem 4.3. The linear equation (4.20) is symmetric (self-adjoint) and positive definite for the variable $n^{k+1}$.

Proof. Considering the $L^{2}$-inner products of $n^{k}$ and 1 , we have

$$
\int_{\Omega} n^{k+1} d x=\int_{\Omega} n^{k} d x=\cdots=\int_{\Omega} n^{0} d x
$$

Set $\alpha_{n}^{0}=1 /|\Omega| \int_{\Omega} n^{0} d x$ and let

$$
\beta^{\mu}=\frac{1}{|\Omega|} \int_{\Omega} \mu^{k+1 / 2} d x, \quad \hat{n}^{k+1}=n^{k+1}-\alpha_{n}^{0}, \quad \hat{\mu}^{k+1 / 2}=\mu^{k+1 / 2}-\beta^{\mu} .
$$

Then, $n^{k+1}$ and $\mu^{k+1 / 2}$ are the solutions of the equations

$$
\begin{aligned}
& \frac{n}{\delta t}-\Delta \mu=\frac{n^{k}}{\delta t} \\
& \mu=-\frac{c}{2} \Delta n-\frac{c}{2} \Delta n^{k}+H\left(n^{*}\right) W\left(n^{k}\right)+\frac{H^{2}\left(n^{*}\right)}{4}\left(n-n^{k}\right)-\beta^{\mu}
\end{aligned}
$$


under the constrain $\int_{\Omega} u d x=0$.

Applying the operator $-\Delta^{-1}$ to the Eq. (4.21) and taking into account the relation (4.22), one obtains

$$
-\frac{\Delta^{-1} n}{\delta t}-\frac{c}{2} \Delta n+\frac{H^{2}\left(n^{*}\right)}{4} n=-\frac{\Delta^{-1} n^{k}}{\delta t}+\frac{c}{2} \Delta n^{k}-W\left(n^{k}\right) H\left(n^{*}\right)+\frac{H^{2}\left(n^{*}\right)}{4} n^{k} .
$$

This equation can be written as $\hat{L} n=\hat{f}$, where

$$
\hat{L} n=-\frac{\Delta^{-1} n}{\delta t}-\frac{c}{2} \Delta n+\frac{H^{2}\left(n^{*}\right)}{4} n-\beta^{\mu} .
$$

Let $u, v$ satisfy the condition $\int_{\Omega} u d x=\int_{\Omega} v d x=0$. Then

$$
\begin{aligned}
(\hat{L} u, v) & =\left(-\frac{\Delta^{-1} u}{\delta t}, v\right)-\frac{c}{2}(\Delta u, v)+\left(\frac{H^{2}\left(n^{*}\right)}{4} u, v\right)-\left(\beta^{\mu}, v\right), \\
& =\left(-\frac{\Delta^{-1} u}{\delta t}, v\right)-\frac{c}{2}(\Delta u, v)+\left(\frac{H^{2}\left(n^{*}\right)}{4} u, v\right), \\
(\hat{L} v, u) & =\left(-\frac{\Delta^{-1} v}{\delta t}, u\right)-\frac{c}{2}(\Delta v, u)+\left(\frac{H^{2}\left(n^{*}\right)}{4} v, u\right)-\left(\beta^{\mu}, u\right) \\
& =\left(-\frac{\Delta^{-1} v}{\delta t}, u\right)-\frac{c}{2}(\Delta v, u)+\left(\frac{H^{2}\left(n^{*}\right)}{4} v, u\right),
\end{aligned}
$$

so that $(\hat{L} u, v)=(\hat{L} v, u)$. Hence, $\hat{L}$ is a self-adjoint operator and for $v=u$ the inner products above take the form

$$
\begin{aligned}
(\hat{L} u, u) & =\left(-\frac{\Delta^{-1} u}{\delta t}, u\right)-\frac{c}{2}(\Delta u, u)+\left(\frac{H^{2}\left(n^{*}\right)}{4} u, u\right)-\left(\beta^{\mu}, u\right) \\
& =\frac{1}{\delta t}\|u\|_{H^{-1}}^{2}+\frac{c}{2}\|\nabla u\|^{2}+\frac{1}{4}\left\|H\left(n^{*}\right) u\right\|^{2} \geq 0 .
\end{aligned}
$$

As before, we note that the inequality becomes an equality if and only if $u=0$.

Remark 4.2. The Lax-Milgram theorem can again be used to show the unique solvability of the system (4.20).

Theorem 4.4. The second order scheme (4.15)-(4.17) is unconditionally energy stable - i.e. it satisfies the discrete energy dissipation law

$$
F_{2 s t}^{k+1}=F_{2 s t}^{k}-\delta t\left\|\nabla \mu^{k+1}\right\|^{2},
$$

where

$$
F_{2 s t}^{k}=\frac{c}{2}\left\|\nabla n^{k}\right\|^{2}+\left\|W^{k}\right\|^{2}
$$


Proof. Considering the $L^{2}$-inner products of (4.15) and $\mu^{k+1},(4.16)$ and $-\left(n^{k+1}-n^{k}\right) /$ $\delta t,(4.17)$ and $2 W^{k+1 / 2}$, we obtain

$$
\begin{aligned}
\left(\frac{n^{k+1}-n^{k}}{\delta t}, \mu^{k+1 / 2}\right)= & -\left\|\nabla \mu^{k+1 / 2}\right\|^{2} \\
\left(\mu^{k+1 / 2},-\frac{n^{k+1}-n^{k}}{\delta t}\right)= & -\frac{c}{2 \delta t}\left(\left\|\nabla n^{k+1}\right\|^{2}-\left\|\nabla n^{k}\right\|^{2}\right) \\
& -\left(W^{k+1 / 2} H\left(n^{*}\right), \frac{n^{k+1}-n^{k}}{\delta t}\right), \\
\left(\frac{W^{k+1}-W^{k}}{\delta t}, 2 W^{k+1 / 2}\right) & =\left(H\left(n^{*}\right) \frac{n^{k+1}-n^{k}}{\delta t}, W^{k+1 / 2}\right) .
\end{aligned}
$$

It follows from (4.23), (4.24) and (4.25) that

$$
\frac{c}{2}\left(\left\|\nabla n^{k+1}\right\|^{2}-\left\|\nabla n^{k}\right\|^{2}\right)+\left(\left\|W^{k+1}\right\|^{2}-\left\|W^{k}\right\|^{2}\right)=-\delta t\left\|\nabla \mu^{k+1 / 2}\right\|^{2}
$$

which implies

$$
\frac{c}{2}\left\|\nabla n^{k+1}\right\|^{2}+\left\|W^{k+1}\right\|^{2}=\frac{c}{2}\left\|\nabla n^{k}\right\|^{2}+\left\|W^{k}\right\|^{2}-\delta t\left\|\nabla \mu^{k+1 / 2}\right\|^{2},
$$

thus finishing the proof.

Remark 4.3. For the second order scheme, the discrete energy dissipation law is an equation, not an inequality.

Remark 4.4. The energy stability is represented in the terms of an alternate energy functional (3.2) instead the original representation (2.1).

Remark 4.5. The system used in the construction of numerical schemes is not equivalent to the initial problem. Therefore, the corresponding convergence analysis is not trivial and will be carried out in future studies.

\section{Numerical Results}

In this section, we apply the proposed numerical schemes to the fourth order parabolic equation with Peng-Robinson equation of state. The substance selected for the comparison with the results of [38] is isobutane (nC4). The critical properties and the normal boiling point of this substance are provided in Table 1 . The parameters $a(T), b$ and $c(T)$ are calculated by formulas (A.1) and (A.2). All the following numerical results are obtained for the domain $\Omega=[0, L]^{2}$ with $L=2 \times 10^{-8}$ meters. 
Table 1: Critical properties - cf. [17, Table 3.1], and $\omega, m$ for isobutane (nC4).

\begin{tabular}{||c|c|c|c|c||}
\hline symbol & $T_{c}, K$ & $P_{c}$ & $\omega$ & $\mathrm{m}$ \\
\hline$n \mathrm{C}_{4}$ & 425.18 & $3.797 \mathrm{MPa}$ & 0.1990 & 0.6709 \\
\hline
\end{tabular}

\subsection{Numerical accuracy}

Here, we present the convergence rate in time for the first order scheme (denoted by IEQ-1st) and the second order Crank-Nicolson scheme (denoted by IEQ-CN2). The initial condition shows the liquid density of isobutane under a saturated pressure condition at temperature $350 \mathrm{~K}$ in the subdomain $[0.3 \mathrm{~L}, 0.7 \mathrm{~L}]^{2}$ with the same temperature isobutane gas occupying the rest of the domain. Table 2 shows how the Cauchy difference varies at the final time. Thus new schemes achieve the convergence rate, which asymptotically corresponds the expected orders in time.

Table 2: Cauchy convergence test at time $=3.0 \mathrm{E}-18$ with central finite difference on the uniform mesh $256 \times 256$ used for spatial discretisation.

\begin{tabular}{||c|c|c|c|c|c||}
\hline \multicolumn{3}{|c|}{ Scheme } & \multicolumn{2}{c|}{ IEQ-1st } & \multicolumn{2}{c||}{ IEQ-CN2 } \\
\hline Coarse time steps & Fine time steps & $L_{2}$ error & Conv. Rate & $L_{2}$ error & Conv. Rate \\
\hline 10 & 20 & $2.252 \mathrm{E}-6$ & - & $3.902 \mathrm{E}-6$ & - \\
\hline 20 & 40 & $1.253 \mathrm{E}-6$ & 0.85 & $3.437 \mathrm{E}-6$ & 0.18 \\
\hline 40 & 80 & $6.588 \mathrm{E}-7$ & 0.93 & $1.804 \mathrm{E}-6$ & 0.93 \\
\hline 80 & 160 & $3.417 \mathrm{E}-7$ & 0.95 & $4.760 \mathrm{E}-7$ & 1.92 \\
\hline 160 & 320 & $1.757 \mathrm{E}-7$ & 0.96 & $1.141 \mathrm{E}-7$ & 2.06 \\
\hline
\end{tabular}

\subsection{Spatial distribution of molar density and other chemical properties}

Let us first consider the above problem in the case of a one droplet. The surface tension contribution to the Helmholtz free energy density $f_{\text {int } f \text { Tens }}$ and the thermodynamic pressure $p_{0}$ are defied as in [38] - i.e.

$$
\begin{aligned}
& f_{\text {intf Tens }}=2 f_{\nabla}(n)=c \nabla n \cdot \nabla n \\
& p_{0}=n\left(\frac{\partial f_{0}}{\partial n}\right)-f_{0}=\frac{n R T}{1-b n}-\frac{n^{2} a(T)}{1+2 b n-b^{2} n^{2}}
\end{aligned}
$$

The evolution history and steady state obtained from the above schemes are very close, so that each one can illustrate the same phenomenon. Here we use the solution obtained by the IEQ-CN2 scheme to find all the parameters required. The domain $\Omega$ is discretised by $256 \times 256$ rectangular mesh.

We study the one droplet situation - i.e. the liquid of the isobutane density under saturated pressure condition at the temperature $350 \mathrm{~K}$ is concentrated in the subdomain $[0.3 \mathrm{~L}, 0.7 \mathrm{~L}]^{2}$, with the saturated isobutane gas occupying the rest of the domain. 

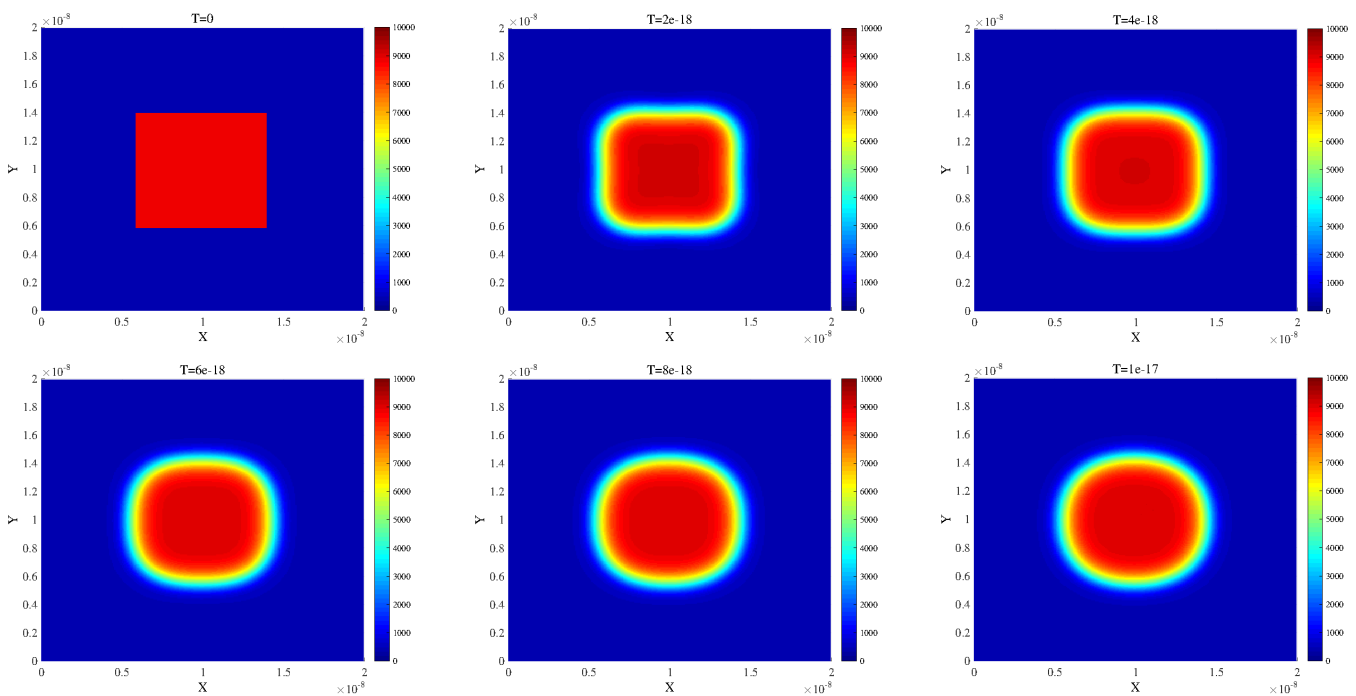

Figure 1: Simulated dynamical evolution of molar density distribution for single droplet. Snapshots are taken at the time $t=0,2 \mathrm{E}-18,4 \mathrm{E}-18,6 \mathrm{E}-18,8 \mathrm{E}-18,1 \mathrm{E}-17$.
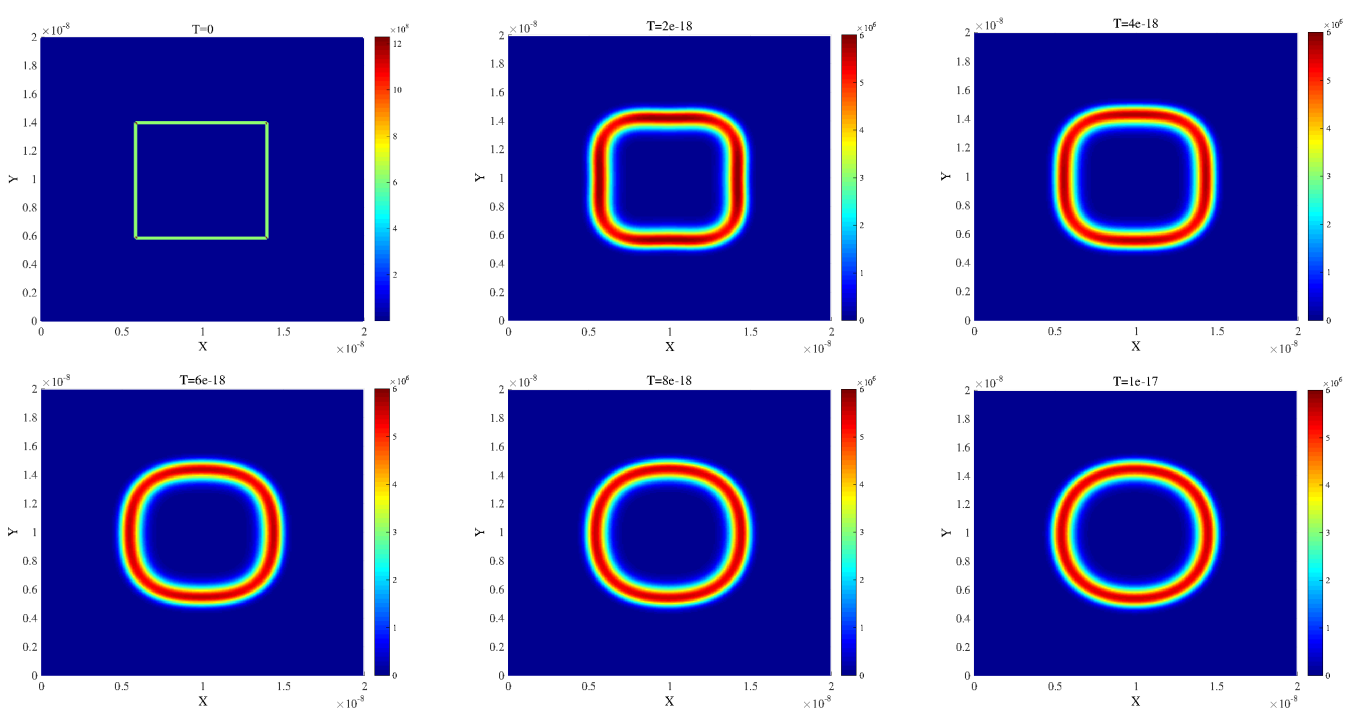

Figure 2: Simulated dynamical evolution of surface tension for single droplet. Snapshots are taken at the time $t=0,2 \mathrm{E}-18,4 \mathrm{E}-18,6 \mathrm{E}-18,8 \mathrm{E}-18,1 \mathrm{E}-17$.

Fig. 1 displays the molar density of isobutane $\left(\mathrm{nC}_{4}\right)$ for the single droplet case at different times. Initially, the liquid droplet has the form of a square, the corners of which round out as with the time, finally becoming a perfect circle. The IEQ-CN2 scheme for the fourth order parabolic equation provides similar molar density evolution history and final state as the convex-splitting scheme in [38]. The simulation of the surface tension contribution to the Helmholtz free energy density is presented in Fig. 2. In addition, clear dissipative trend of the total Helmholtz free energy during the whole evolution history and the mass 

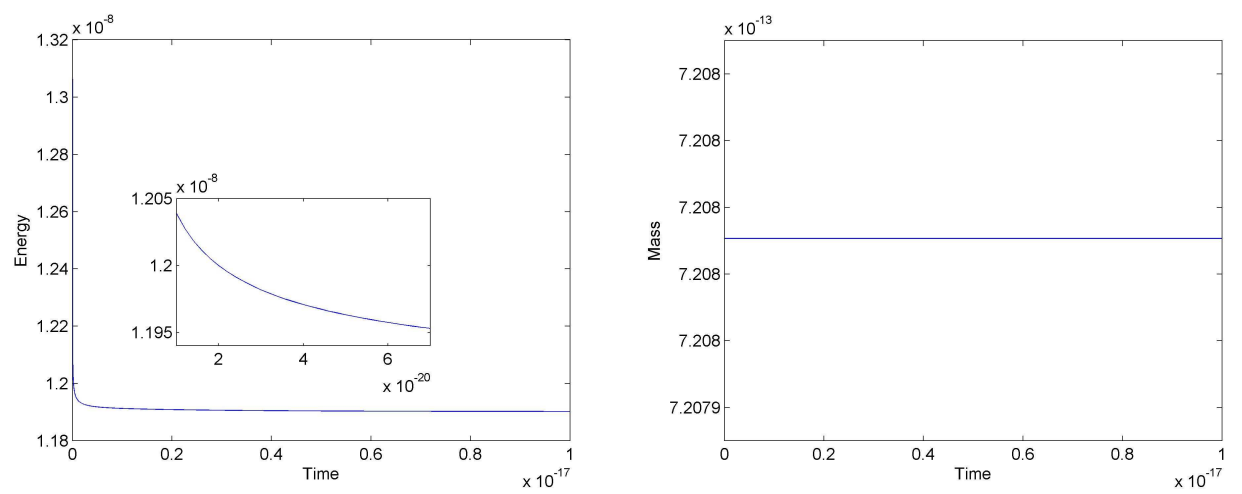

Figure 3: Evolution of total energy and mass. Single droplet case, simulated by IEQ-CN2 scheme, $\delta t$ $=2.0 \mathrm{E}-21$.
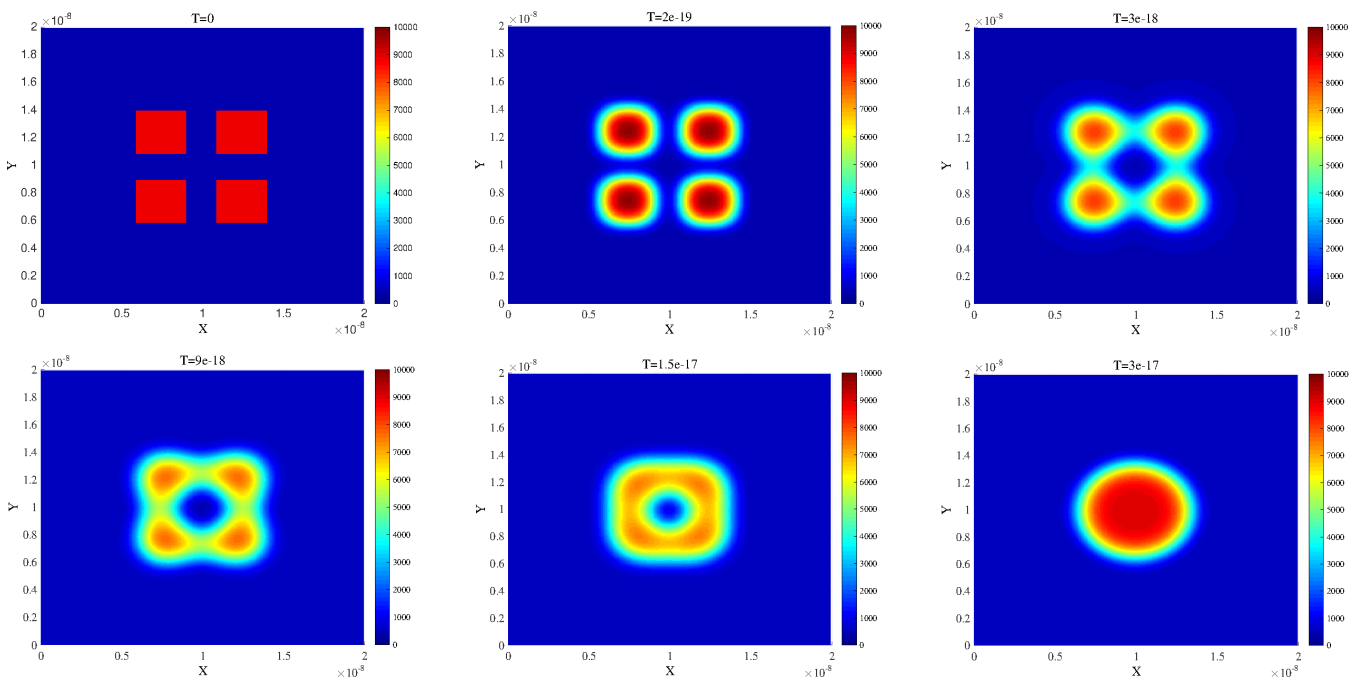

Figure 4: Simulated dynamical evolution of molar density distribution for four droplets. Snapshots are taken at the time $t=0,2 \mathrm{E}-19,3 \mathrm{E}-18,9 \mathrm{E}-18,1.5 \mathrm{E}-17,3 \mathrm{E}-17$.

are demonstrated in Fig. 3. We point out that energy decreases monotonically, whereas mass is accurately maintained all the time.

We now use the IEQ-CN2 scheme to describe the solution evolution in the four droplet case under a specific initial condition. The molar density distribution at different time points and the energy evolution during the whole process are displayed in Fig. 4. Initially, there are four commensurate square bubbles $\{(0.3 L, 0.45 L),(0.55 L, 0.7 L)\}^{2}$ symmetrically located with respect to the center of the domain with gas-liquid interface around every single bubble at the initial time. At the early stage, the bubbles round out separately. Then nearly circular bubbles touch neighboring ones. The contact areas gradually increase and the whole construction transforms into the unit circle. Finally, single ring becomes a circular droplet at the domain center. Fig. 5 shows the surface tension at the same times. The total energy monotonically decreases and then rapidly decays after the shape of the liquid 

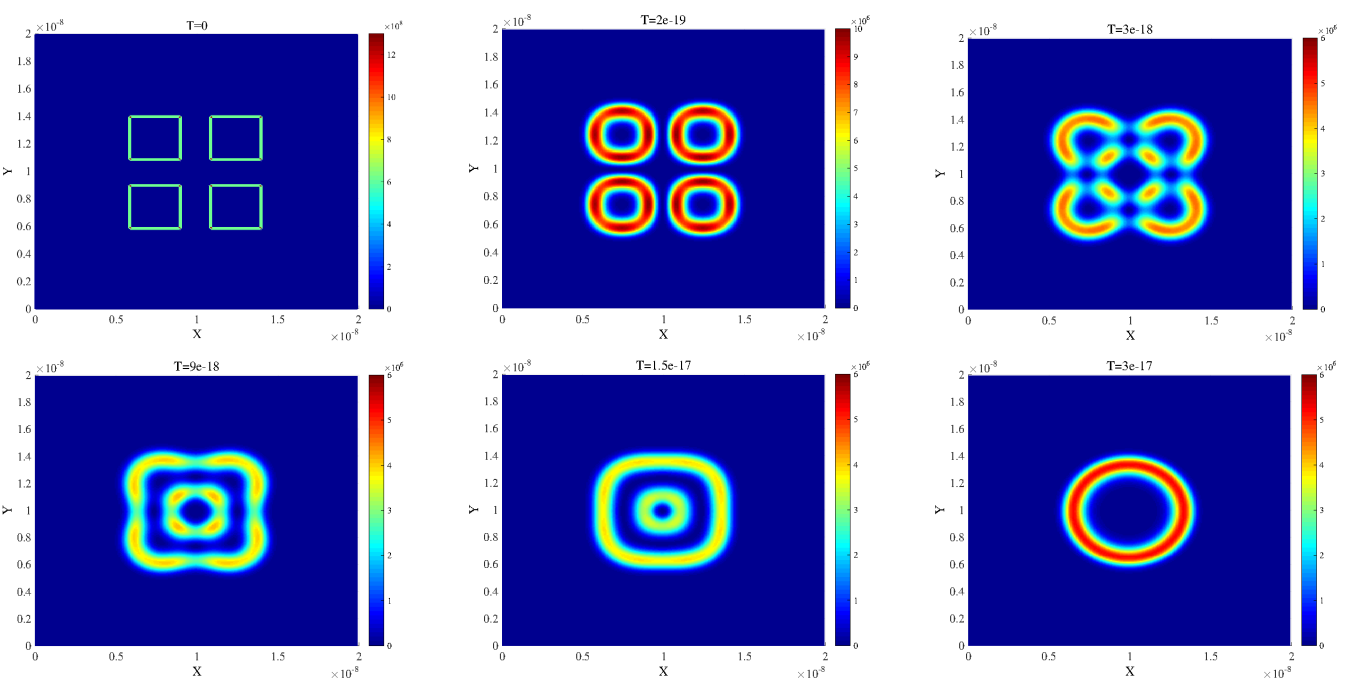

Figure 5: Simulated dynamical evolution of surface tension for four droplets. Snapshots are taken at the time $t=0,2 \mathrm{E}-19,3 \mathrm{E}-18,9 \mathrm{E}-18,1.5 \mathrm{E}-17,3 \mathrm{E}-17$.
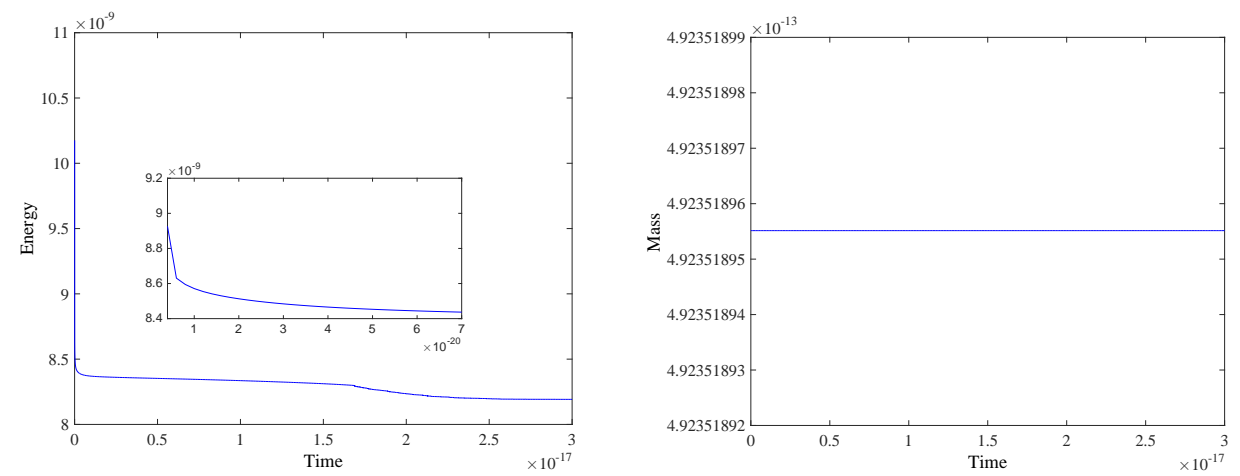

Figure 6: Evolution of total energy and mass for four droplets, simulated by the IEQ-CN2 scheme, $\delta t$ $=2.0 \mathrm{E}-21$.

bubbles changes dramatically - cf. Fig. 6, whereas mass is accurately maintained all the time.

\subsection{Calculation of interface tension and verification against Young-Laplace equation}

The surface tension $\sigma$ is defined as the net contractive force per unit length with a unit of $N / m$ mechanically or the work for creating a unit area of interface with a unit of $\mathrm{J} / \mathrm{m}^{2}$. Assuming that for the given system the surface tension is spatially constant within the interface, one can represent it in the form - cf. [17,38]:

$$
\sigma=\frac{\partial F}{\partial A}=\frac{F(n)-F_{0}\left(n_{b u l k}\right)}{A}
$$




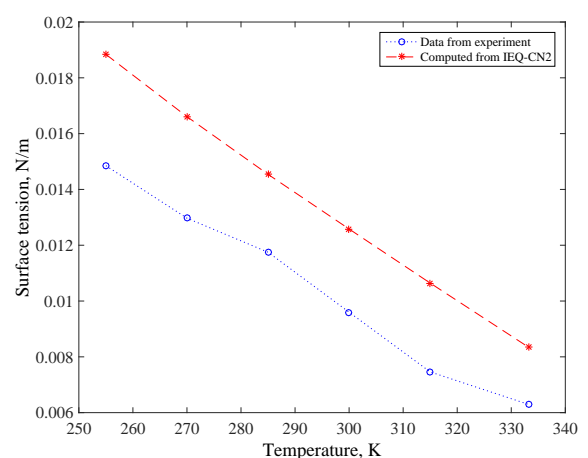

(a)

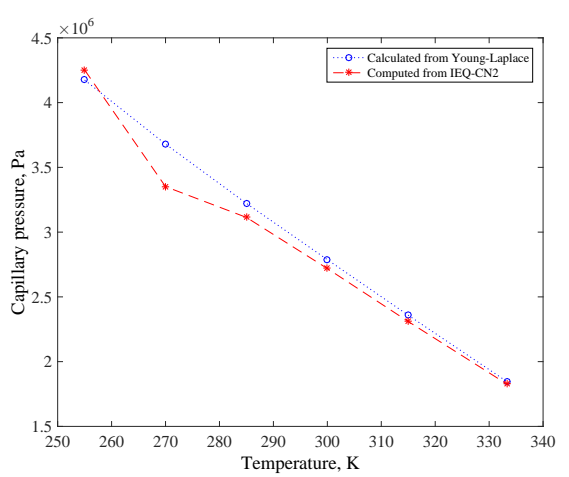

(b)

Figure 7: Numerical and Experimental Results. Left: Surface tension. Right: Capillary pressure.

The numerator in the Eq. (5.1) evaluates the contribution of the surface tension to the total free energy at the equilibrium state. The volume of the liquid droplet is assumed to be constant all the time and droplet becomes a circle at the steady state. The radius and the length of the droplet are, respectively, $r=2 \times 10^{-8} \times(0.16 / \pi)^{1 / 2}=4.514 \times 10^{-9}$ and $A=2 \pi \times 4.514 \times 10^{-9}$ meters. Fig. 7(a) shows the surface tension determined by our approximation method. The results are compared with the experimental data in [32, Table 2] and they are sufficiently close from the engineering point of view.

The pressure is calculated by the well-known Young-Laplace equation $P_{c}=P_{\text {liquid }}-$ $P_{\text {gas }}=\sigma / r-$ cf. Refs. $[17,38]$. The pressure of the liquid drop $P_{\text {liquid }}$ and the gas region $P_{\text {gas }}$ are, respectively, evaluated at the grid points $(128,128)$ and $(38,38)$. The difference between them $p_{0}=P_{\text {liquid }}-P_{\text {gas }}$ is the capillary pressure. On the other hand, the capillary pressure given by the Young-Laplace equation is $p=\sigma / r$. The results obtained by these methods in the temperature range $250 \mathrm{~K}-350 \mathrm{~K}$ are displayed in Fig. 7 (b).

\section{Conclusion}

We developed first and second order numerical schemes for the fourth order parabolic equation with Peng-Robinson equation of state, which are based on recently proposed invariant energy quadratisation method. Both schemes are linear, unconditionally energy stable and uniquely solvable. Moreover, since the reduced linear systems are symmetric and positive definite, their solutions can be efficiently found. Numerical results demonstrate the good performance of the schemes.

\section{Acknowledgments}

The authors are very grateful to Professor Lili Ju for numerous valuable discussions. We also would like to thank the anonymous reviewers for careful reading of our manuscript and for constructive comments greatly improved this work. 
H.L. is partially supported by the National Natural Science Foundation of China (Grants Nos. 11401350, 11471196) and by the China Scholarship Council (Grant No. 201608370010), Q.P. is supported by the Fundamental Research Funds for the Central Universities, the Research Funds of Renmin University of China (Grant No. 17XNLF03), by China Postdoctoral Sustentation Fund (Grant No. 2017M611085) and by the National Natural Science Foundation of China (Grant No. 11701562) and Z.X. is partially supported by National Natural Science Foundation of China (Grant No. 11571365).

\section{Appendix. Parameters in the Peng-Robinson Equation of State}

The parameters $a(T)$ and $b$ can be evaluated as follows

$$
a(T)=0.45724 \frac{R^{2} T_{c}^{2}}{P_{c}}\left(1+m\left(1-\sqrt{\frac{T}{T_{c}}}\right)\right)^{2}, \quad b=0.07780 \frac{R T_{c}}{P_{c}},
$$

where $T_{c}$ and $P_{c}$ are, respectively, the critical temperature and the critical pressure of the corresponding substance. The parameter $m$ is related to the acentric parameter $\omega-$ viz.

$$
\begin{aligned}
& m=0.37464+1.54226 \omega-0.26992 \omega^{2}, \quad \omega \leq 0.49, \\
& m=0.379642+1.485030 \omega-0.164423 \omega^{2}+0.016666 \omega^{3}, \quad \omega>0.49 .
\end{aligned}
$$

The coefficient $c$ in (2.1) is the influence parameter of the pure substance and has the form

$$
c=a b^{2 / 3}\left(m_{1}^{c}\left(1-\frac{T}{T_{c}}\right)+m_{2}^{c}\right)
$$

where

$$
m_{1}^{c}=-\frac{10^{-16}}{1.2326+1.3757 \omega}, \quad m_{2}^{c}=\frac{10^{-16}}{0.9051+1.5410 \omega} .
$$

\section{References}

[1] D. Anderson, G.B. McFadden and A. Wheeler, Diffuse-interface methods in fluid mechanics, Annu. Rev. Fluid Mech. 30, 139-165 (1998).

[2] A. Baskaran, Z. Hu, J.S. Lowengrub, C. Wang, S. Wise and P. Zhou, Energy stable and efficient finite-difference nonlinear multigrid schemes for the modified phase field crystal equation, J. Comput. Phys. 250, 270-292 (2013).

[3] J. Bosch, C. Kahle and M. Stoll, Preconditioning of a coupled Cahn-Hilliard Navier-Stokes system, Commun. Comput. Phys. 23, 603-628 (2018).

[4] B. Breure and C. Peters, Modeling of the surface tension of pure components and mixtures using the density gradient theory combined with a theoretically derived influence parameter correlation, Fluid Phase Equilibr. 334, 189-196 (2012).

[5] J.W. Cahn, Free energy of a nonuniform system. II. Thermodynamic basis, J. Chem. Phys. 30, 1121-1124 (1959).

[6] J.W. Cahn. On spinodal decomposition, Acta Metall. 9, 795-801 (1961). 
[7] J.W. Cahn and J.E. Hilliard, Free energy of a nonuniform system. I. Interfacial free energy, J. Chem. Phys. 28, 258-267 (1958).

[8] W. Chen, S. Conde, C.Wang, X. Wang and S. Wise, A linear energy stable scheme for a thin film model without slope selection, J. Sci. Comput. 52, 546-562 (2012).

[9] W. Chen, C. Wang, X. Wang and S. Wise. A linear iteration algorithm for a second-order energy stable scheme for a thin film model without slope selection, J. Sci. Comput. 59, 574-601 (2014).

[10] M. Copetti and C. Elliott, Numerical analysis of the Cahn-Hilliard equation with a logarithmic free energy, Numer. Math. 63, 39-65 (1992).

[11] S.M. Cox and P.C. Matthews, Exponential time differencing for stiff systems, J. Comput. Phys. 176, 430-455 (2002).

[12] H.T. Davis, Statical mechanics of phases, interfaces, and thin films, VCH (1996).

[13] W. E, Nonlinear continuum theory of smetic-A liquid crystals, Arch. Ration. Mech. Anal. 137, 159-175 (1997).

[14] C.M. Elliott and H. Garcke, On the Cahn-Hilliard equation with degenerate mobility, SIAM J. Math. Anal. 27, 404-423 (1996).

[15] D.J. Eyre, Unconditionally gradient stable time marching the Cahn-Hilliard equation, MRS Proceedings, 529, 39-46 (1998).

[16] X. Fan, J. Kou, Z. Qiao and S. Sun, Modeling pore-scale oil-gas systems using gradient theory with Peng-Robinson equation of state, Procedia Comput. Sci. 80, 1364-1373 (2016).

[17] A. Firoozabadi, Thermodynamics of hydrocarbon reservoirs, McGraw-Hill, New York (1999).

[18] S. Gu, H. Zhang and Z. Zhang, An energy-stable finite-difference scheme for the binary fluidsurfactant system, J. Comput. Phys. 270, 416-431 (2014).

[19] F. Guillén-González and G. Tierra, On linear schemes for a Cahn-Hilliard diffuse interface model, J. Comput. Phys. 234, 140-171 (2013).

[20] R. Guo and Y. Xu, A high order adaptive time-stepping strategy and local discontinuous Galerkin method for the modified phase field crystal equation, Commun. Comput. Phys. 24, 123-151 (2018).

[21] M.E. Gurtin. Generalized Ginzburg-Landau and Cahn-Hilliard equations based on a microforce balance, Phys. D 92, 178-192 (1996).

[22] M.E. Gurtin, D. Polignone and J. Viňals. Two-phase binary fluids and immiscible fluids described by an order parameter, Math. Model. Meth. Appl. Sci. 6, 815-831 (1996).

[23] K.B. Haugen, L. Rongy and A. Firoozabadi, Mixing from fickian diffusion and natural convection in binary non-equilibrium fluid phases, AIChE J. 58, 1336-1345 (2012).

[24] Z. Hu, S. Wise, C. Wang and J.S. Lowengrub, Stable and efficient finite-difference nonlinearmultigrid schemes for the phase field crystal equation, J. Comput. Phys. 228, 5323-5339 (2009).

[25] L. Ju, J. Zhang and Q. Du, Fast and accurate algorithms for simulating coarsening dynamics of Cahn-Hilliard equations, Comput. Mater. Sci. 108, 272-282 (2015).

[26] J. Kou and S. Sun, An adaptive finite element method for simulating surface tension with the gradient theory of fluid interfaces, J. Comput. Appl. Math. 255, 593-604 (2014).

[27] J. Kou and S. Sun. Numerical methods for a multicomponent two-phase interface model with geometric mean influence parameters, SIAM J. Sci. Comput. 37, B543-B569 (2015).

[28] J. Kou and S. Sun, Multi-scale diffuse interface modeling of multi-component two-phase flow with partial miscibility, J. Comput. Phys. 318, 349-372 (2016).

[29] J. Kou, S. Sun and X. Wang. Efficient numerical methods for simulating surface tension of multicomponent mixtures with the gradient theory of fluid interfaces, Comput. Method. Appl. Mech. Engin. 292, 92-106 (2015).

[30] H. Li, L. Ju, C. Zhang and Q. Peng, Unconditionally energy stable linear schemes for a diffuse interface model with Peng-Robinson equation of state, J. Sci. Comput. 75, 993-1015 (2018). 
[31] D. Li, Z. Qiao and T. Tang, Characterizing the stabilization size for semi-implicit Fourier-Spectral method to phase field equations, SIAM J. Numer. Anal. 54, 1653-1681 (2016).

[32] H. Lin and Y. Duan, Surface tension measurements of propane ( $r-290)$ and isobutane $(r-600 a)$ from (253 to 333) K, J. Chem. Eng. Data 48, 1360-1363 (2003).

[33] A. Onuki, Phase transition dynamics, Cambridge University Press (2002).

[34] D.Y. Peng and D.B. Robinson.A new two-constant equation of state, Ind. Eng. Chem. Fundam. 15(1), 59-64 (1976).

[35] Q. Peng, A convex-splitting scheme for a diffuse interface model with Peng-Robinson equation of state, Adv. Appl. Math. Mech. 9, 1162-1188 (2017).

[36] Q. Peng, Z. Qiao and S. Sun, Stability and convergence analysis of second-order schemes for a diffuse interface model with Peng-Robinson equation of state, J. Comput. Math. 35, 737-765 (2017).

[37] Q. Peng, Z. Zhang and H. Zhang, The phase transition model for heat-shrinkable thermosensitive hydrogels based on interaction energy, Commun. Comput. Phys. 17, 594-614 (2015).

[38] Z. Qiao and S. Sun, Two-phase fluid simulation using a diffuse interface model with PengRobinson equation of state, SIAM J. Sci. Comput. 36(4), B708-B728 (2014).

[39] Z. Qiao, Z. Sun and Z. Zhang, The stability and convergence of two linearized finite difference schemes for the nonlinear epitaxial growth model, Numer. Methods Partial Differential Equations 28(6), 1893-1915 (2012).

[40] Z. Qiao, Z. Sun and Z. Zhang, Stability and convergence of second-order schemes for the nonlinear epitaxial growth model without slope selection, Math. Comput. 84, 653-674 (2015).

[41] D.B. Robinson, D. Peng and S.Y. Chung, The development of the Peng-Robinson equation and its application to phase equilibrium in a system containing methanol, Fluid Phase Equilibr. 24, 25-41 (1985).

[42] J.S. Rowlinson, Translation of J.D. van der Waals. "The thermodynamik theory of capillarity under the hypothesis of a continuous variation of density", J. Statist. Phys. 20, 197-244 (1979).

[43] J. Shen, C. Wang, X. Wang and S. Wise, Second-order convex splitting schemes for gradient flows with Ehrlich-Schwoebel type energy: application to thin film epitaxy, SIAM J. Numer. Anal. 50, 105-125 (2012).

[44] J. Shen and X. Yang, Numerical approximations of Allen-Cahn and Cahn-Hilliard equations, Discret. Contin. Dyn. Syst. A 28, 1669-1691 (2010).

[45] P. Vignal, L. Dalcin, D.L. Brown, N. Collier and V.M. Calo, An energy-stable convex splitting for the phase-field crystal equation, Comput. Structu. 158, 355-368 (2015).

[46] C. Wang, X. Wang and S. Wise, Unconditionally stable schemes for equations of thin film epitaxy, Discret. Contin. Dyn. Syst. A 28, 405-423 (2010).

[47] C. Wang and S. Wise. An energy stable and convergent finite-difference scheme for the modified phase field crystal equation, SIAM J. Numer. Anal. 49, 945-969 (2011).

[48] X. Wang, L. Ju and Q. Du, Efficient and stable exponential time differencing Runge-Kutta methods for phase field elastic bending energy models, J. Comput. Phys. 316, 21-38 (2016).

[49] S. Wise, C. Wang and J.S. Lowengrub, An energy-stable and convergent finite-difference scheme for the phase field crystal equation, SIAM J. Numer. Anal. 47, 2269-2288 (2009).

[50] D. Wu and H. Zhang. Numerical investigation of the growth kinetics for macromolecular microsphere composite hydrogel based on the TDGL equation, J. Theor. Comput. Chem. 15, 1650064 (2016).

[51] C. Xu and T. Tang, Stability analysis of large time-stepping methods for epitaxial growth models, SIAM J. Numer. Anal. 44, 1759-1779 (2006).

[52] Y. Yan, W. Chen, C. Wang and S. Wise, A second-order energy stable BDF numerical scheme for the Cahn-Hilliard equation, Commun. Comput. Phys. 23, 572-602 (2018). 
[53] X. Yang and D. Han, Linearly first- and second-order, unconditionally energy stable schemes for the phase field crystal model, J. Comput. Phys. 330, 1116-1134 (2017).

[54] X. Yang and L. Ju, Accurate and unconditionally energy stable linear schemes for the binary fluid-surfactant phase field model, Comput. Meth. Appl. Mech. Eng. 318, 1005-1029 (2017).

[55] X. Yang and L. Ju, Efficient linear schemes with unconditionally energy stability for the phase field elastic bending energy model, Comput. Meth. Appl. Mech. Eng. 315, 691-712 (2017).

[56] J. Zhao, Q. Wang and X. Yang, Numerical approximations for a phase field dendritic crystal growth model based on the invariant energy quadratization approach, Int. J. Numer. Meth. Eng. 110, 279-300 (2017). 